\title{
Effects of Difference in the Guiding System on the Results of Thrombectomy
}

Akikazu Nakamura, Keigo Shigeta, Takanori Hayakawa, Hiroshi Yatsushige, Kyoko Sumiyoshi, Toshiya Momose, Masaya Enomoto, Shin Sato, Jiro Aoyama, Hiroyuki Masaoka, and Yoshio Takasato

Objective: The effects of difference in the guiding system on the results of stent thrombectomy were investigated.

Methods: Of the patients who underwent intracranial thrombectomy using a stent retriever in 2015 and 2016, those with lesions in the internal carotid artery (ICA) or the horizontal (M1) segment of the middle cerebral artery were divided into a group treated with a balloon guide catheter (BGC) alone and a group treated with a combination of BGC + distal access (aspiration) catheter (DAC), and 1) the time from puncture to recanalization, 2) number of passes, 3) frequency of occurrence of embolization to new/distal territory (ENT/EDT), and 4) percentage of complete recanalization were compared.

Results: The subjects were 17 patients consisting of 6 in the BGC group and 11 in the BGC + DAC group. The results concerning the above items were 1) 80.0 vs. $46.7 \mathrm{~min}(p=0.03), 2) 2.8$ vs. 1.5 times $(p=0.03), 3) 50 \%$ vs. $0 \%(p=0.03)$, and 4$) 66.7 \%$ vs. $90.9 \%(p=0.52)$, respectively.

Conclusion: In the BGC + DAC group, the time until recanalization was shorter, the number of passes was fewer, and the frequency of ENT/EDT was lower, suggesting that more favorable results may be obtained by BGC + DAC.

Keywords stent retriever, solitaire, delivery system, Penumbra

\section{Introduction}

Currently, the trend is that thrombectomy is recommended for emergent large vessel occlusion (ELVO) following intravenous thrombolysis with recombinant tissue plasminogen activator.

According to the guidelines for the early management of patients with acute ischemic stroke regarding endovascular treatment (updated in 2015), in using a stent retriever, the use of a balloon guide catheter (BGC) or distal access catheter (DAC) is recommended rather than a cervical

Department of Neurosurgery, National Hospital Organization Disaster Medical Center, Tokyo, Japan

Received: February 15, 2017; Accepted: June 26, 2017 Corresponding author: Akikazu Nakamura. Department of Neurosurgery, National Hospital Organization Disaster Medical Center, 2-9-1-2307 Osaki, Shinagawa, Tokyo 141-0032, Japan Email: akikazu0326@gmail.com

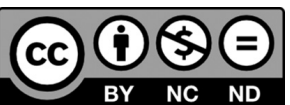

This work is licensed under a Creative Commons Attribution-NonCommercialNoDerivatives International License.

(C)2017 The Japanese Society for Neuroendovascular Therapy guide catheter alone (Class IIa, Level of Evidence: C). The guidelines also mention that future studies should examine which stent delivery systems provide the highest recanalization rate with the lowest risk for embolization to new/distal territory (ENT/EDT). $\left.{ }^{1}\right)$

The objective of this study was to characterize a stent delivery system that achieves the highest recanalization rate with the lowest risk of ENT/EDT.

\section{Subjects and Methods}

Of the patients who underwent thrombectomy for ELVO at our hospital in 2015 and 2016, those treated for occlusion of the internal carotid artery (ICA) or the horizontal segment (M1) of the middle cerebral artery using a stent retriever were analyzed. All types of occlusion were included in the study without setting inclusion criteria regarding the cause of occlusion (cardiogenic, atheromatous, etc.). The subjects were divided into those in which BGC was used alone as the stent delivery system (BGC group) and those in which $\mathrm{BGC}$ and $\mathrm{DAC}$ were used in combination (BGC + DAC group). There was no rule as to 
which stent delivery system should be used, and the decision was made by the judgment of the experts in endovascular surgery who performed the procedure (physician A or B). The time from puncture to recanalization, number of passes through the lesion, percentage of patients with ENT/ EDT, and percentage of patients who had thrombolysis in cerebral infarction (TICI) 3 were compared between the two groups. Statistical analyses were performed using the t-test or Fisher's exact test at the $p<0.05$ level of significance.

\section{Intracranial thrombectomy procedure}

In the BGC group, after a microcatheter was passed through the lesion, the stent retriever was deployed, the balloon was inflated in the cervical ICA, and the stent was retrieved while manually applying a negative pressure to the BGC.

In the BGC + DAC group, Penumbra 5MAX ACE (Penumbra Inc., Alameda, CA, USA) was used as the DAC. After it was inserted as far as possible, a microcatheter was immediately passed through the lesion, and the stent retriever was deployed. Then, the Penumbra was advanced to a point nearest the thrombus, and the microcatheter was removed. An aspiration tube was attached to the Penumbra, and the stent retriever was removed together with the Penumbra by aspirating with MAX Pump (Penumbra Inc.). At this time, a negative pressure was also applied manually to the BGC. Figure 1 shows the thrombectomy method in each group.

\section{Results}

Between January 2015 and July 2016, thrombectomy using a stent retriever was performed for embolism of the ICA and $\mathrm{M} 1$ in 17 patients consisting of 6 in the BGC group and 11 in the BGC + DAC group. Patient background is shown in Table 1. There was no clear difference between the two groups in 1) the site of occlusion, 2) age, or 3 ) $\operatorname{sex}(p=$ $0.16, p=0.46, p=1$, respectively). The percentage of patients with cardiogenic embolism was $66.7 \%$ and $72.7 \%$ in the BGC and BGC + DAC groups, respectively, with no significant difference $(p=1)$.

The results are shown in Table 2. The time from groin puncture to recanalization was 80 minutes in the BGC group but 46.7 minutes in the BGC + DAC group and was significantly shorter in the latter group $(\mathrm{p}=0.03)$. The number of passes through the occluded area needed until recanalization was 2.8 in the $\mathrm{BGC}$ group but 1.5 in the

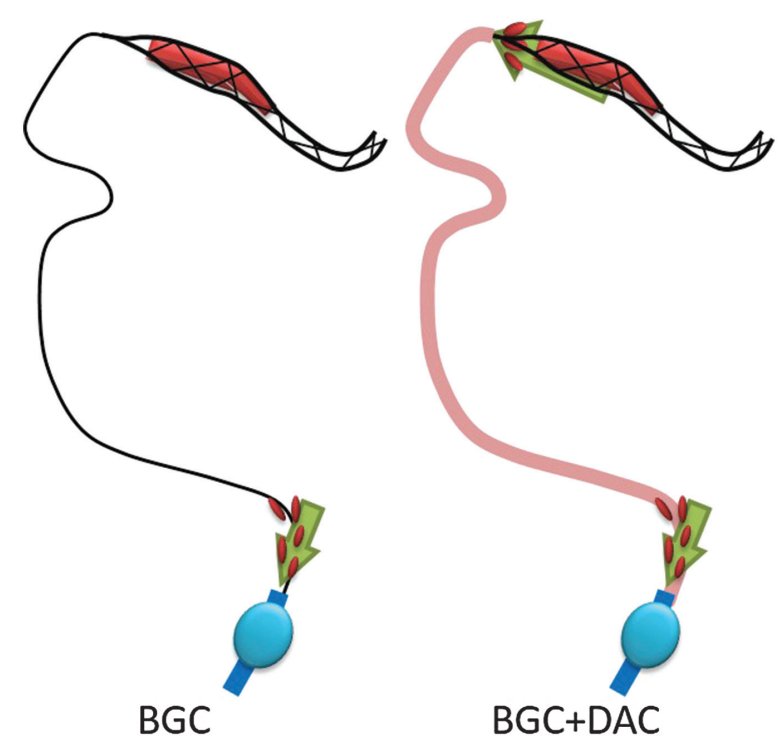

Fig. 1 Diagrams of the BGC and BGC + DAC groups. In the BGC + DAC group, the intermediate catheter was inserted to a point closest to the stent, and aspiration was also made through the intermediate catheter. BGC: balloon guide catheter; DAC: distal access catheter

BGC + DAC group, and recanalization was achieved by significantly fewer passes in the latter group $(p=0.03)$. ENT/EDT also occurred significantly less frequently in the BGC + DAC group $(p=0.03)$. No significant difference was observed in the TICI3 recanalization rate between the two groups $(\mathrm{p}=0.52)$.

The percentages of the procedures performed by the two physicians (A and $\mathrm{B}$ ) were examined in the two groups to confirm that the differences in the therapeutic results were not due to the difference in the physician's skill. The percentages of the procedures performed by physician A were $50 \%$ and $81.8 \%$ in the BGC and BGC + DAC groups, respectively, with no significant difference $(\mathrm{p}=0.28)$.

\section{Discussion}

Percutaneous intracranial thrombectomy has been demonstrated to be effective as a treatment for acute intracranial artery occlusion of the anterior circulation and is recommended by the guidelines. As a guide catheter that navigates the stent retriever to the lesion, BGC or DAC rather than a balloonless cervical guide catheter is recommended. ${ }^{1)}$ In 2013, Deshaies et al. ${ }^{2)}$ reported two cases treated by the combined stent-aspiration technique using a DAC and recommended it as an effective method for thrombectomy as the DAC provides satisfactory support to the stent, the treatment can be promptly switched to aspiration if stenting is 
Table 1 Patient background

\begin{tabular}{lcccc} 
& & BGC & BGC + DAC & $p$ \\
Site of occlusion & ICA & $4(66.7)$ & $3(27.3)$ & 0.16 \\
$\mathrm{n}(\%)$ & $\mathrm{M} 1$ & $2(33.3)$ & $8(72.7)$ & \\
Age & & $69.0 \pm 10.4$ & $74.8 \pm 16.8$ & 0.46 \\
Females n (\%) & & $3(50)$ & $6(54.5)$ & 1 \\
\hline
\end{tabular}

BGC: balloon guide catheter; DAC: distal access (aspiration) catheter; ICA: internal carotid artery

\begin{tabular}{lcccc} 
Table 2 Results & & & \\
& $\begin{array}{c}\text { Puncture-recanalization } \\
(\min )\end{array}$ & No. of passes & $\begin{array}{c}\text { ENT/EDT } \\
(\%)\end{array}$ & TICl 3 (\%) \\
BGC alone & $80.0 \pm 40.3$ & $2.8 \pm 1.8$ & $50 \%$ & $66.7 \%$ \\
BGC + DAC & $46.7 \pm 16.8$ & $1.5 \pm 0.5$ & $0 \%$ & $90.9 \%$ \\
p & 0.03 & 0.03 & 0.03 & 0.52 \\
\hline
\end{tabular}

BGC: balloon guide catheter; DAC: distal access (aspiration) catheter; ENT/EDT: embolization to new/distal territory; $\mathrm{TICl}$ : thrombolysis in cerebral infarction

found to be ineffective, and a high thrombectomy effect can be obtained by aspiration from a site close to the stent. There have been reports of a few in vitro studies concerning which delivery system provides the highest recanalization rate with the lowest incidence of ENT/EDT.,3) In this study, we reviewed past cases treated at our institution and compared the therapeutic results between groups treated with BGC alone and in combination with DAC. The time from puncture to recanalization was significantly shorter in the BGC + DAC group ( $\mathrm{p}=0.03)$. This is considered to be related to the fewer number of passes through the lesion and lower frequency of ENT/EDT in the BGC + DAC group. Despite an in vitro study, it was reported that the incidence of ENT/EDT was lower when BGC or the combined stent-aspiration technique was used compared with a simple guide catheter alone. ${ }^{3)}$ Satisfactory therapeutic results can also be expected with BGC alone, but, in our study, the results were even more favorable when BGC was used in combination with DAC, probably because more thrombus could be retrieved due to the synergistic effect of the stent retriever + aspiration under occlusion of the anterograde blood flow with a balloon.

Since validation of the therapeutic results in this study may be insufficient due to the small number of subjects, they are compared with previous reports from large volume centers. Humphries et al. reported 105 cases of thrombectomy using the stent retriever + aspiration. This technique resembles our procedure using $\mathrm{BGC}+\mathrm{DAC}$. The mean number of passes was 2.3 , time from puncture to recanalization was 57.3 minutes, TICI3 recanalization rate was $43.8 \%$, and incidence of embolism at other sites was $5.7 \%$. ${ }^{5}$ There were also two reports of stent retriever + aspiration in 2016.6,7) While simple comparison is impossible, the results in the $\mathrm{BGC}+\mathrm{DAC}$ group of our study were comparable to the results of these reports except for the time from puncture to recanalization reported by McTaggart et al.7) (14 minutes) (Table 2).

However, 80 minutes was required from puncture to recanalization in the BGC group. It far exceeded recent target times, probably because the percentage of patients with ICA occlusion was higher in the BGC group (66.7\%) although the difference was not significant. The volume of thrombus may have been larger, and the treatment may have been difficult, in many patients with ICA occlusion. Despite the significant difference in time until recanalization, no significant difference was observed in the TICI3 recanalization rate between the two groups. In this study, more time was needed until recanalization, more passes through the lesion were necessary, and ENT occurred more frequently, in the BGC group, but complete recanalization could be eventually achieved similarly to the BGC + DAC group.

A limitation of this study was that it was a single-center retrospective study involving a small number of patients. While there was no statistically significant difference in patient background, there was a undeniable impression that there were differences in patient background such as a higher frequency of ICA occlusion in the BGC group. Therefore, further accumulation of cases is necessary.

\section{Conclusion}

In the $\mathrm{BGC}+\mathrm{DAC}$ group, the time until recanalization was significantly shorter, the number of passes was fewer, 
and the frequency of ENT/EDT was lower. The possibility that BGC + DAC improves the therapeutic results was suggested. Since this study was a single-center retrospective study, further accumulation of cases and examination of the outcomes and complication rate are considered necessary.

\section{Disclosure Statement}

The first author and all of the coauthors have no conflicts of interest to disclose.

\section{References}

1) William J. Powers, Colin P. Derdeyn, José Biller, et al: AHA/ ASA guideline 2015 AHA/ASA focused update of the 2013 guidelines for the early management of patients with acute ischemic stroke regarding endovascular treatment. A guideline for healthcare professionals from the American heart association/American stroke association. Stroke 2017; 48: doi: https://doi.org/10.1161/STR.0000000000000074.
2) Deshaies EM: Tri-axial system using the solitaire-FR and penumbra aspiration microcatheter for acute mechanical thrombectomy. J Clin Neurosci 2013; 20: 1303-1305.

3) Mokin M, Ionita CN, Nagesh SV, et al: Primary stentriever versus combined stentriever plus aspiration thrombectomy approaches: in vitro stroke model comparison. J Neurointerv Surg 2015; 7: 453-457.

4) Mokin M, Setlur Nagesh SV, Ionita $\mathrm{CN}$, et al: Comparison of modern stroke thrombectomy approaches using an in vitro cerebrovascular occlusion model. AJNR Am J Neuroradiol 2015; 36: 547-551.

5) Humphries W, Hoit D, Doss VT, et al: Distal aspiration with retrievable stent assisted thrombectomy for the treatment of acute ischemic stroke. J Neurointerv Surg 2015; 7: 90-94.

6) Massari F, Henninger N, Lozano JD, et al: ARTS (aspirationretriever technique for stroke): initial clinical experience. Interv Neuroradiol 2016; 22: 325-332.

7) McTaggart RA, Tung EL, Yaghi S, et al: Continuous aspiration prior to intracranial vascular embolectomy (CAPTIVE): a technique which improves outcomes. J Neurointerv Surg 2016; 16 December doi: 10.1136/neurintsurg-2016-012838. 